\title{
Estimación indirecta de la función renal y mortalidad por insuficiencia cardíaca: buscando el mejor predictor
}

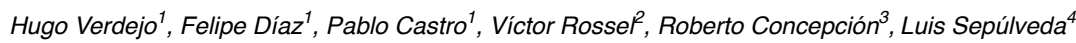 \\ 1 División de Enfermedades Cardiovasculares, Pontificia Universidad Católica de Chile. \\ 2 Hospital del Tórax. \\ 3 Hospital DIPRECA. \\ 4 Hospital J.J. Aguirre, Universidad de Chile
}

Antecedentes: Uno de los principales predictores de eventos adversos en pacientes con insuficiencia cardíaca (IC) es el deterioro de función renal luego de una hospitalización. Estudios recientes han cuestionado la utilidad de la fórmula de Cockroft-Gault (CG) para estimar la función renal en estos pacientes.

Objetivo: Evaluar la utilidad de distintos modelos para determinación indirecta de función renal como predictores de mortalidad alejada en sujetos con IC.

Método: Estudio descriptivo analítico. Se incorporaron en forma consecutiva pacientes admitidos de 14 hospitales chilenos con el diagnóstico de IC descompensada entre enero 2002 y julio 2012. Las características de los pacientes se compararon mediante $t$ de Student o chi cuadrado según correspondía. En base a la creatininemia al alta se estimó la tasa de filtración glomerular mediante las formulas CG, MDRD-4 y CKD-Epi. El acuerdo entre los resultados de cada fórmula se analizó mediante kappa de acuerdo para clasificación en estadios de función renal y según el método de Bland-Altman. Los pacientes se clasificaron por cuartiles según la estimación de cada fórmula; la sobrevida se analizó con el método de Kaplan-Meier y la capacidad de cada fórmula para predecir eventos mediante un modelo de riesgo proporcional de Cox. La mortalidad al fin del seguimiento se determinó por la base de datos del Servicio Nacional de Registro Civil e Identificación.
Resultados: Se incluyeron 1584 pacientes, 45,3\% de sexo femenino. La edad promedio fue de 70,5 \pm 20 años. La creatinina al ingreso fue $1,63 \pm 1,48 \mathrm{mg} / \mathrm{dL}$ y al egreso de $1,59 \pm 1,41 \mathrm{mg} / \mathrm{dL}(\mathrm{p}=\mathrm{NS}$ ). Todos los modelos estimaron un porcentaje similar de población con falla renal al momento del alta $(\mathrm{Cl} \mathrm{Crea}<60 \mathrm{ml} / \mathrm{min} / 1.73 \mathrm{~m} 2)$ : CG 57,3\%, MDRD-4 54,9\% y CKD-Epi $54,9 \%$. El clearance de creatinina estimado por CG $(59,4 \pm 30,4$ $\mathrm{mL} / \mathrm{min} / 1,74$ ) fue mayor al estimado por CKD-Epi o MDRD-4 (54,2 $\pm 25,1$ y $57,8 \pm 27 \mathrm{~mL} / \mathrm{min} / 1,73 \mathrm{~m} 2)$. De manera significativa, tanto CRD-Epi como MDRD-4 lograron identificar correctamente un subgrupo de pacientes con baja mortalidad alejada (CKD-Epi cuartil 3 y 4 , OR 0,5 IC95\% 0,35-0,72; MDRD-4 cuartil 3 y 4, OR 0,56 IC95\% 0,34-0,80). La fórmula de CG no permitió la identificación de subgrupos con menor mortalidad. El análisis de curva ROC sugiere un punto de corte de 60 $\mathrm{mL} / \mathrm{min} / 1.73 \mathrm{~m} 2$ para CKD-Epi (Sensibilidad 0,63; Especificidad: 0,43 ) o para MDRD-4 (Sensibilidad 0,60; Especificidad 0,45).

Conclusión: La estimación de la tasa de filtración glomerular (TFG) mediante MDRD-4 y CKD-Epi permiten identificar una población de sujetos con bajo riesgo de mortalidad alejada. La implementación de estas formulas simples permitirán caracterizar mejor el perfil de riesgo de los pacientes con IC al momento del alta. 


\section{Estimation of renal function and mortality in congestive heart failure: a search for the best indicator}

Background. One of the best predictors of adverse events in patients with congestive heart failure (CHF) is the deterioration of renal function following hospitalization. Recent studies have questioned the usefulness of the Cockroft-Gault (CG) formula to estimate renal function in these patients.

Aim: To evaluate the usefulness of different indirect methods for estimation of renal function in the prediction of late mortality in patients with CHF.

Method: Consecutive patients admitted for CHF in 14 different hospitals from January 2002 and July 2012 were analyzed. Student's t or Chi square were used as appropriate for statistical comparisons. The serum creatinine level at the time of discharge was used to estimate glomerular filtration rate (GFR) from 3 different formulae: CG, MDRD-4 and CKD-Epi. Agreement among methods for different assignment to different stages of renal failure was evaluated by the "kappa" statistics and the Bland- Altmann method. Survival according to the estimation from each formula was compared by the log-rank statistics on KaplanMeier's survival curves. The ability of each formula to predict adverse events was evaluated by a Cox proportional hazards method. Mortality was obtained from de National Identification Registry.

Results: 1548 patients were included, $45.3 \%$ of them females. Mean age was 70.5 years (SD 20). Creatinine level was $1,63 \pm 1,48 \mathrm{mg} / \mathrm{dL}$ at admission and $1,59 \pm 1,41 \mathrm{mg} / \mathrm{dL}$ at discharge $(\mathrm{p}=\mathrm{NS})$. The proportion of patients with renal failure (creatinine clearance $<60 \mathrm{ml} / \mathrm{min} / 1.73 \mathrm{~m} 2$ ) was similar for all methods (CG: 57.3\%, MDRD-4 54.9\% and CKDEpi $54.9 \%)$. Creatinine clearance by CG $(59,4 \pm 30,4$ $\mathrm{mL} / \mathrm{min} / 1,73 \mathrm{~m} 2$ ) was higher than that estimated by CKD-EPI $(54,2 \pm 25,1 / 1.73 \mathrm{~m} 2)$ or MDRD-4 (57,8 \pm 27 $\mathrm{mL} / \mathrm{min} / 1,73 \mathrm{~m} 2$ ). Both CKD-Epi and MDMD-4 correctly identified a subgroup of patients with lower late mortality (CKD-Epi quartiles 3 and 4, OR 0,5 IC95\% 0,35-0,72; MDRD-4 quartiles 3 and 4, OR 0,56 IC95\% 0,34-0,80). The CG formula did not identified subgroups with lower mortality rate. A cut point of $60 \mathrm{~mL} / \mathrm{min} / 1.73 \mathrm{~m} 2$ (calculated by ROC analysis) determined a sensitivity of 0.63 and specificity of 0.43 for the CKD-Epi estimation and corresponding values of 0.60 and 0.45 for the MDRD- 4 method

Conclusion: GFR estimated by the MDRD-4 or the CKD-Epi methods allow the identification of a group of patients with a lower late mortality rate among patients with $\mathrm{CHF}$ in a simple, clinically useful way.

Keywords: Congestive heart failure, Renal insufficiency, Mortality, Epidemiology. 


\section{Introducción:}

La insuficiencia cardíaca (IC) es una condición de prevalencia creciente asociada a una elevada mortalidad y morbilidad. Su evolución se caracteriza por un deterioro progresivo de la capacidad funcional, habitualmente asociado a múltiples rehospitalizaciones debido a descompensación. Dentro de los predictores más importantes de eventos en pacientes con IC se encuentra el deterioro de la función renal después de una hospitalización. Si bien en los últimos años la interpretación de los cambios en función renal luego de una hospitalización por IC han sido reevaluados, la evidencia parece coincidir en que un deterioro significativo de la función renal en pacientes con IC se asocia a un marcado incremento del riesgo de muerte y rehospitalización ${ }^{1}$.

En la práctica clínica diaria suele recurrirse a la determinación de la creatinina plasmática como un marcador de la función renal. Sin embargo, esta medición presenta variaciones importantes en relación a edad, sexo, etnia y dieta ${ }^{2}$, a lo que se suma la limitación planteada por la relación exponencial entre creatinina y la tasa de filtración glomerular TFG: son necesarias caídas de más del $50 \%$ en la TFG para elevar la creatinina plasmática por sobre el nivel de referencia ${ }^{3}$. Dado que la determinación directa de la TFG es engorrosa y cara, se han desarrollado fórmulas para la estimación de la TFG en base a los valores de creatinina plasmática, utilizando como factores correctores variables demográficas y antropométricas. De estas fórmulas, la más conocida y empleada es la de Cockroft-Gault(CG), de amplia difusión en la clínica. Esta ecuación, publicada en 1976, se desarrolló para inferir el clearance de creatinina como un estimado de la TFG, a partir de una población de 23 adultos, mayoritariamente hombres ${ }^{4}$. Sin embargo, y pese su utilidad como aproximación rápida a la TFG, la fórmula CG se asocia a sesgos importantes, en particular en individuos con IC, subestimando la TFG cuando esta se encuentra por encima de $60 \mathrm{ml} / \mathrm{min} / 1,73 \mathrm{~m} 2$ y sobrestimándola cuando se encuentra por debajo de 30 $\mathrm{ml} / \mathrm{min} / 1,73 \mathrm{~m} 2$. Aún cuando esta limitación puede ser parcialmente corregida empleando el peso ideal y no el peso real del paciente al momento de calcular la TFG, la fórmula de $\mathrm{CG}$ se considera actualmente inadecuada para la estimación de función renal en pacientes con IC. Para corregir esta falencia se han postulado otros modelos para la estimación indirecta de la función renal. La ecuación MDRD-4 (Modification of Diet in Renal Disease) corrige en gran medida el sesgo de la fórmula de CG, con lo que ha ganado aceptación creciente en ambientes clínicos. La ecuación MDRD-4 se desarrolló con el objetivo de mejo- rar la precisión de la fórmula CG estimando TFG y no Cl Crea; para tal efecto, se estudió la TFG de 1070 adultos de ambos sexos con algún grado de falla renal. Aún cuando la estimación de TFG por MDRD-4 es más confiable que la obtenida con $\mathrm{CG}^{5}$, el hecho que su fórmula haya sido derivada de una población con falla renal hace que la estimación de TFG sobre $60-90 \mathrm{ml} / \mathrm{min} / 1,73 \mathrm{~m} 2$ mediante MDRD-4 suele ser poco fiable ${ }^{6}$.

Recientemente, el grupo CKD-Epi (Chronic Kidney Disease Epidemiology Collaboration) publicó una nueva ecuación desarrollada a partir de una gran muestra de 8.254 individuos $^{7}$. La fórmula CKD-Epi incluye ajustes por sexo, edad y raza, lo que permite obtener resultados más precisos, en particular para valores de TFG superiores a $60 \mathrm{ml} / \mathrm{min} / 1,73 \mathrm{~m} 2$, por lo cual algunos autores han preconizado su incorporación a la práctica clínica habitual ${ }^{8}$. Sin embargo, hasta el momento, no existen estudios que hayan comparado la utilidad de las tres fórmulas en la evaluación del perfil de riesgo de pacientes con IC en nuestro medio.

\section{Métodos:}

Estudio prospectivo. Desde enero de 2002 a Julio del 2009, en 14 centros chilenos, se recolectó información prospectiva de 1164 pacientes hospitalizados por IC CF III o IV mediante una ficha electrónica específicamente diseñada para este fin. El criterio diagnóstico fue: síntomas de IC y evidencias objetivas de disfunción cardíaca objetivada en el ecocardiograma o la radiografía de tórax; en casos dudosos se consideró la respuesta al tratamiento dirigido a la IC. Se excluyeron todos los pacientes en los cuales el motivo principal de hospitalización no fue la IC y aquellos pacientes que estando previamente asintomáticos y sin antecedente de IC previa se presentaron en shock cardiogénico secundario a un cuadro coronario agudo. De igual manera, se excluyeron para este trabajo aquellos pacientes con terapia de sustitución renal crónica al momento del ingreso al hospital. Se registraron características demográficas, comorbilidad, previsión, nivel educacional, antecedentes de hospitalizaciones previas por IC, etiología de la cardiopatía de base, factores de descompensación de la hospitalización actual, hallazgos del electrocardiograma y ecocardiograma, incluida la fracción de eyección de ventrículo izquierdo (FEVI). Se consignó el tratamiento al ingreso, alta y evolución intrahospitalaria. Las historias clínicas correspondientes, incluyendo radiografías de tórax, exámenes de laboratorio, incluyendo creatinina de ingreso, alta y ecocardiogramas, fueron revisadas por el equipo investigador de cada centro. 
Para cada uno de los pacientes incluidos se calculó la TFG según la creatinina plasmática al momento del alta según las fórmulas detalladas en la Tabla 1. Los pacientes se clasificaron en estadios de función renal según las recomen-

Tabla 1. Ecuaciones para la estimación de la tasa de filtrado glomerular

Cockroft-Gault (Clearance de creatinina estimado)

$(140-$ Edad $){ }^{*}$ Peso

72 * Creatinina $*(0,85$ si es mujer $)$

MDRD-4 (Tasa de filtración glomerular estimada)

186 * (Creatinina) $-1,154$ *(Edad) $-0,203 *(0,742$ si es mujer $) *(1,210$ si raza negra $)$

CKD-Epi (Tasa de filtración glomerular estimada para etnia blanca y otras)

Mujeres

Si creatinina $<62: 144$ * $\left(\left[\right.\right.$ Creatinina/88,4/0,7)] ${ }^{-0,329}$ * 0,993 edad

Si creatinina $>62: 144$ * $\left(\left[\text { Creatinina/88,4/0,7)] }{ }^{-1,209}\right)^{*} 0,993\right.$ edad

Hombres

Si creatinina $<80: 141^{*}\left(\left[\right.\right.$ Creatinina/88,4/0,9)] $\left.{ }^{-0,411}\right) * 0,993$ edad

Si creatinina $>80: 141$ * $([$ Creatinina/88,4/0,9)] $-1,209) * 0,993$ edad

daciones de la K/DOQUI-National Kidney Foundation? . Para efectos de análisis, se definió IC con función sistólica conservada como aquellos pacientes con el diagnóstico de IC y FEVI igual o superior a 50\%. Hemoconcentración al alta se definió como la presencia de un incremento en el valor del hematocrito de tres o más puntos porcentuales en ausencia de historia de transfusiones. La mortalidad al fin del seguimiento se determinó por la base de datos del Servicio Nacional de Registro Civil e Identificación.

Análisis estadístico: El objetivo primario del estudio fue comparar el valor pronóstico de la TFG estimada según CG, MDRD-4 y CKD-Epi en la mortalidad alejada de pacientes admitidos por IC descompensada. Las características de la población fueron expresadas como media \pm desviación estándar para variables continuas y como porcentaje (frecuencia) para variables categóricas. Dada la naturaleza retrospectiva de los datos en métodos se habla de estudio prospectivo: concordar, y considerando la imposibilidad de contar con una determinación objetiva del TFG al momento del alta, se empleó el método de Bland-Altman para comparar la variabilidad entre las distintas ecuaciones para la estimación de la TFG. La capacidad de reclasificar pacientes se midió con el test kappa de acuerdo, con un límite de 0,05 , de dos colas para significancia estadística. La sobrevida se evaluó mediante método de Kaplan-Meier. El poder predictivo de las distintas ecuaciones se evaluó mediante un modelo de riesgo proporcional de Cox.

\section{Resultados}

Características demográficas: Entre enero 2008 y julio de 2010 se incluyeron 1570 pacientes en el estudio, 44,1\% de sexo femenino. La edad media era de 70,2 $\pm 13,4$ años. Un $80,9 \%$ de los sujetos tenía antecedentes de HTA y un $36,3 \%$ de diabetes mellitus. Un 33,4\% de los pacientes admitidos fueron clasificados como IC con función sistólica preservada.

La etiología de la IC fue preferentemente hipertensiva $(38,1 \%)$, seguida por cardiopatía coronaria $(25,4 \%)$ y miocardiopatía dilatada (16\%). Las características demográficas y de laboratorio de la población son detalladas en la Tabla 2. Al momento del ingreso, la creatininemia fue de $1,67 \pm 1,5 \mathrm{mg} / \mathrm{dL}$, y presentó una reducción significativa al momento del alta $(1,60 \pm 1,4 \mathrm{mg} / \mathrm{dL}$, t-test para muestras pareadas $\mathrm{p}<0,01)$. Como se aprecia en la Figura 1, CG tendió a sobreestimar levemente la TFG en relación a las otras dos fórmulas, presentando una mayor dispersión en sus resultados.

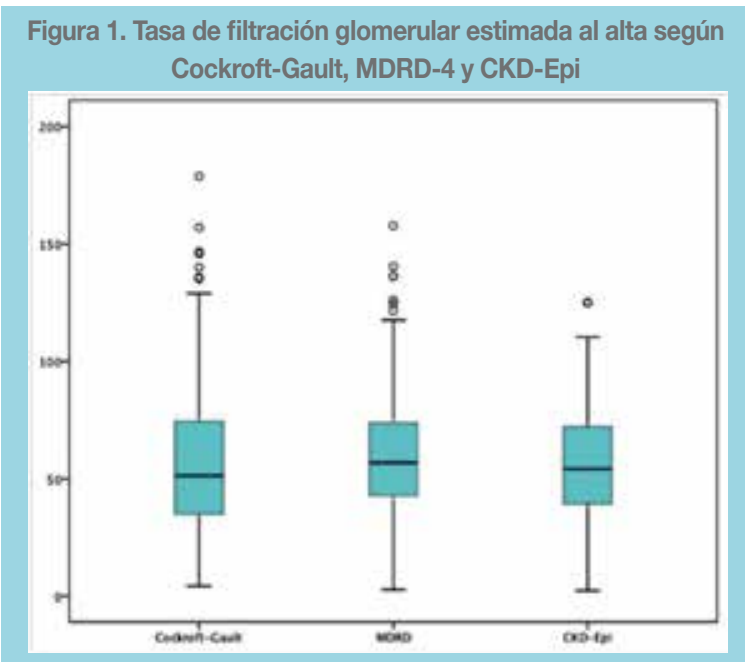

\begin{tabular}{|l|r|}
\hline \begin{tabular}{|l|} 
Tabla 2. Características clínicas de sujetos con \\
Insuficiencia Cardíaca al ingreso
\end{tabular} \\
\hline \multicolumn{2}{|c|}{$\mathrm{N}=1570$} \\
\hline Edad & $70,2 \pm 13,4$ \\
\hline Sexo femenino & $44,1 \%$ \\
\hline HTA & $80,9 \%$ \\
\hline DM & $36,3 \%$ \\
\hline IAM & $26,2 \%$ \\
\hline AVE & $10,8 \%$ \\
\hline FEVI (\%) & $40,9 \pm 17,1$ \\
\hline Hematocrito (\%) & $39,1 \pm 6,8$ \\
\hline Hemoglobina (g/L) & $12,9 \pm 2,3$ \\
\hline Recuento g. blancos (103/mL) & $9,5 \pm 4$ \\
\hline Nitrógeno ureico (mg/dL) & $31,8 \pm 19,5$ \\
\hline Creatinina & $1,63 \pm 1,48$ \\
\hline Na+ (mEg/L) & $137,4 \pm 5,1$ \\
\hline K+ (mEq/L) & $4,3 \pm 0,7$ \\
\hline Albúmina (g/L) & $3,6 \pm 0,6$ \\
\hline Ácido úrico (mg/dL) & $7,3 \pm 3,3$ \\
\hline
\end{tabular}


Variabilidad de las fórmulas para determinación de función renal: La Figura 2 muestra los resultados del análisis de Bland-Altman para CG, MDRD-4 y CKD-Epi. Como es posible observar, la estimación de la TFG por Cockroft presentó una amplia dispersión en relación al valor de referencia establecido por la media de las estimaciones de MDRD-4 y CKD-Epi, lo que se hizo más notable a

Figura 2. Análisis de Bland-Altman para las diferentes fórmulas para estimación de la tasa de filtración glomerular
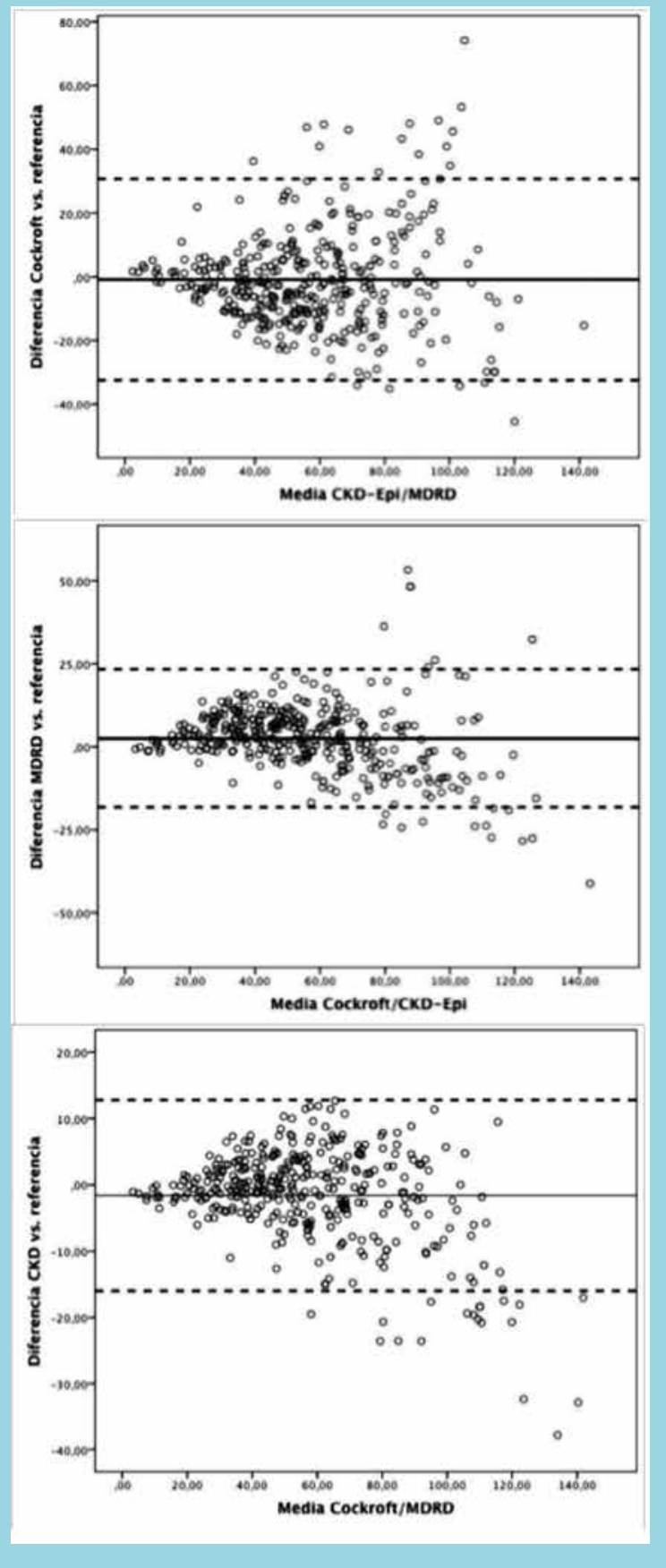

partir de $\mathrm{Cl}$ Crea $>60 \mathrm{ml} / \mathrm{min}$. La ecuación de MDRD-4, por el contrario, tendió a subestimar de manera sistemática la TFG cuando esta excedía el valor crítico de $60 \mathrm{ml} / \mathrm{min}$, aún cuando presento una dispersión de los datos inferior al compararla con CG. La ecuación de CKD-Epi presentó una baja dispersión de los datos hasta TFG 100 mL/min, con una dispersión de los datos inferior a la presentada por CG y comparable a la observada para MDRD-4.

La Tabla 3 muestra la distribución de los pacientes según estadio de función renal de acuerdo de la clasificación propuesta por el KDOQUI. Como es posible apreciar, tanto MDRD-4 como CKD-Epi se asociaron a una significativa reclasificación de los pacientes, identificando un mayor porcentaje de individuos con compromiso leve a moderado de función renal. Como consecuencia de lo anterior, la tasa de acuerdo de CG con MDRD-4 y CKD-Epi fue baja (kappa de acuerdo 0,377 y 0,442, respectivamente, $\mathrm{p}<0,01)$. Por el contrario, la clasificación entre MDRD-4 y CKD-Epi fue altamente coincidente (kappa de acuerdo $0,784, \mathrm{p}<0,01)$.

\section{Análisis de Bland-Altman. Las líneas punteadas representan \pm 2 desviaciones estándar respecto del promedio de las diferencias entre el método evaluado y la medida de referencia}

\begin{tabular}{|c|c|c|c|}
\hline \multicolumn{4}{|c|}{ Estadio (TFG)Cockroft-Gault MDRD-4CKD-Epi } \\
\hline 1 (>90 mL/min) & $14 \%$ & $11,1 \%$ & $8,8 \%$ \\
\hline 2 (60-89 mL/min) & $25,3 \%$ & $33,9 \%$ & $32,6 \%$ \\
\hline 3a (45-59 mL/min) & $18,8 \%$ & $22 \%$ & $21 \%$ \\
\hline 3b (30-45 mL/min) & $22,6 \%$ & $18 \%$ & $18,4 \%$ \\
\hline $4(15-29 \mathrm{~mL} / \mathrm{min})$ & $16,1 \%$ & $9,9 \%$ & $13,1 \%$ \\
\hline 5 (<15 mL/min) & $3,2 \%$ & $5,1 \%$ & $6,1 \%$ \\
\hline
\end{tabular}

Función renal como predictor de mortalidad a largo plazo: Al comparar la sobrevida alejada de cada cuartil de función renal definidos por cada ecuación de estimación, solo MDRD-4 y CKD-Epi fueron capaces de discriminar entre individuos con alto riesgo de mortalidad (cuartil 1 y 2) e individuos con bajo riesgo (cuartil 3 y 4), tal como muestra la Figura 3. La reducción de la TFG por debajo de $60 \mathrm{~mL} /$ min se asoció a una mayor tasa de muertes al año independiente de la fórmula empleada para el cálculo; sin embargo, solo CKD-Epi y MDRD-4 mantuvieron su capacidad de predecir eventos en pacientes con TFG por debajo de 45 $\mathrm{mL} / \mathrm{min}$; la fórmula de Cockroft-Gault entregó resultados inconsistentes en pacientes con mala función renal (Tabla 
Figura 3. Mortalidad alejada según cuartiles de tasa de filtración glomerular estimados por distintas ecuaciones.
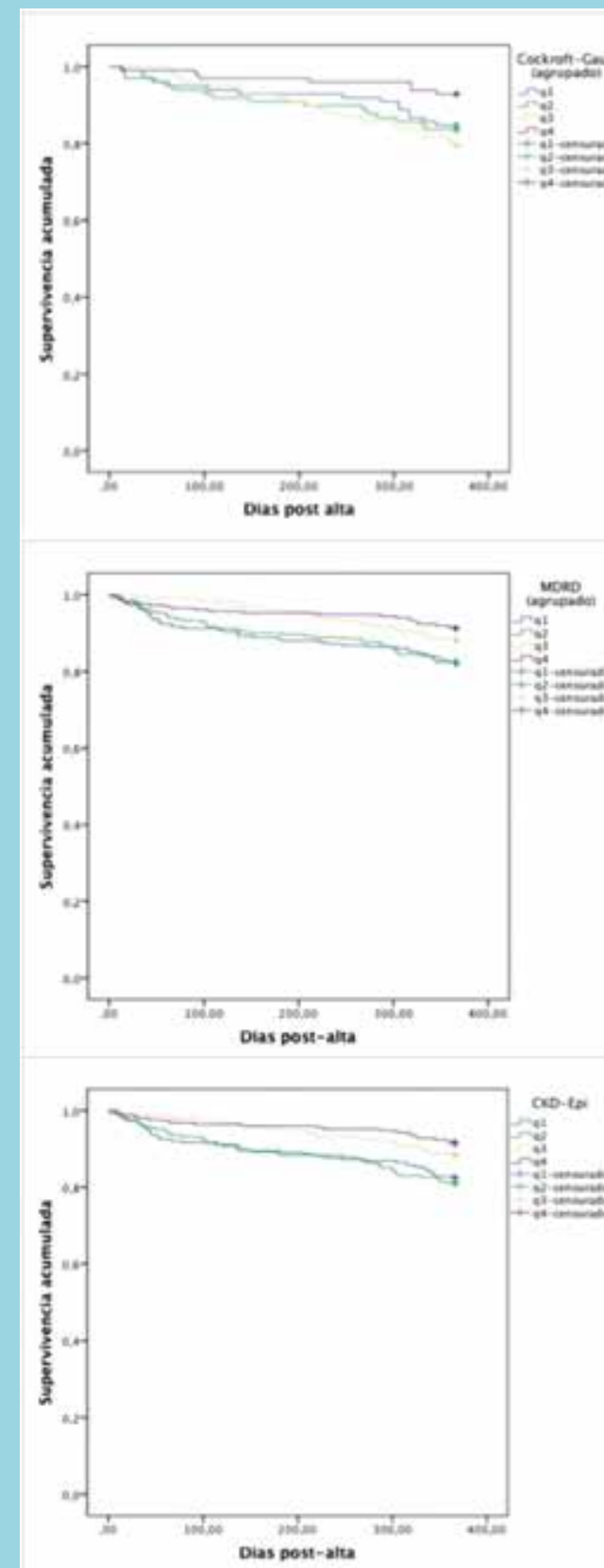

4). Cuando se comparó el poder predictivo de las ecuaciones en pacientes con IC y función sistólica preservada vs. los pacientes con falla sistólica, se evidenció que el deterioro de función renal es un predictor más relevante en la medida que existe compromiso en la función sistólica del VI (Tabla 5). El análisis de curva ROC sugirió un punto de corte de $60 \mathrm{~mL} / \mathrm{min} / 1.73 \mathrm{~m} 2$ para CKD-Epi (Sensibilidad a) Cockroft-Gault log rank test para la comparación q1q2 vs. $q 3 q 4 p=$ N.S. b) MDRD-4 log-rank test para la comparación q1q2 vs. q3q4 p<0,01 c) CKD-Epi log-rank test para la comparación q1q2 vs. $q 3 q 4 p<0,01$.

\begin{tabular}{|c|c|c|c|}
\hline & Cockroft-Gault & MDRD-4 & CKD-Epi \\
\hline \multirow[t]{2}{*}{$\mathrm{TFG}<60 \mathrm{~mL} / \mathrm{min}$} & HR 1,87 & HR 1,82 & HR 1,86 \\
\hline & $(1,04-3,39)^{\star}$ & $(1,27-2,59)^{\star *}$ & $(1,29-2,68)^{\star \star}$ \\
\hline \multirow{2}{*}{$\mathrm{TFG}<45 \mathrm{~mL} / \mathrm{min}$} & HR 1,08 & HR 1,56 & HR 1,70 \\
\hline & $(0,64-1,83)$ & $(1,12-2,18)^{* *}$ & $(1,22-2,37)^{\star *}$ \\
\hline
\end{tabular}

El riesgo de mortalidad a un año post alta se expresa como razón de riesgo (IC95\%). * $p<0,05$; ** $p<0,01$

\begin{tabular}{|c|c|c|c|}
\hline & Cockroft-Gault & MDRD-4 & CKD-Epi \\
\hline \multicolumn{4}{|l|}{ IC con función } \\
\hline \multirow[t]{2}{*}{ sistólica preservada } & HR 1,59 & HR 0,99 & HR 0,92 \\
\hline & $(0,56-4,54)$ & $(0,47-2,11)$ & $(0,43-1,94)$ \\
\hline \multirow[t]{2}{*}{ IC con falla sistólica } & HR 1,59 & HR 1,88 & HR 1,90 \\
\hline & $(0,68-3,69)$ & $(1,08-3,27)^{*}$ & $(1,07-3,36)^{\star}$ \\
\hline
\end{tabular}

El riesgo de mortalidad a un año post alta se expresa como razón de riesgo (IC95\%). * $p<0,05 ;{ }^{* *} p<0,01$

0,63; Especificidad: 0,43) o para MDRD-4 (Sensibilidad 0,60 ; Especificidad 0,45).

Impacto de la hemoconcentración en el significado pronóstico de la función renal: La mayoría de los pacientes admitidos por IC descompensada tienen sobrecarga de volumen. El alivio incompleto de la congestión durante los episodios de descompensación aguda de la enfermedad puede contribuir a la elevada tasa de rehospitalizaciones después de un evento índice. Los datos observacionales parecen apoyar esta hipótesis: los sujetos que logran hemoconcentración luego de una hospitalización por IC descompensada, una medida indirecta de la eficacia de la terapia depletiva, tienen una sobrevida mejor pese a una incidencia aumentada de deterioro de función renal ${ }^{10}$. Empleando como criterio de hemoconcentración un incremento del hematocrito de 3 puntos porcentuales o más, un $28 \%$ de la población en estudio logró los objetivos de hemoconcentración al momento del alta de la hospitalización índice. En estos pacientes, la presencia de deterioro de función renal medida por MDRD-4 o CKD-Epi no se asoció a un peor pronóstico a largo plazo (CKD-Epi $<60 \mathrm{~mL} / \mathrm{min}$ 
HR 2,06 IC95\% 0,53-7,96, $\mathrm{p}=0,24)$; por el contrario, en el grupo que no alcanzó dichos objetivos, el deterioro de función renal mantuvo su significado pronóstico.

Limitaciones del estudio: Dada la naturaleza retrospectiva del análisis, el presente estudio solo comparó tres ecuaciones distintas para la estimación indirecta de función renal, sin incluir un patrón de referencia como la determinación de TFG mediante métodos isotópicos. De igual manera, no se dispone de los datos de cistatina $\mathrm{C}$ o microalbuminuria que podrían contribuir al refinamiento de la TFG estimada por CKD-Epi. De igual manera, el peso al momento del egreso de los pacientes que fue empleado para la estimación de TFG puede no representar el peso seco real del paciente.

\section{Discusión}

En nuestro conocimiento, este es el primer trabajo que valora en nuestro medio la utilidad de tres ecuaciones para la estimación indirecta de la TFG en la predicción de mortalidad alejada luego de una hospitalización índice por IC descompensada. Nuestros resultados son coincidentes con la literatura que sugiere que la incorporación de estimaciones más precisas de la TFG mediante MDRD-4 o CKD-Epi se traducen en una mejor caracterización de los pacientes de riesgo en la población de sujetos con $\mathrm{IC}^{5-11}$.

Pese a la importancia de la función renal en la fisiopatología de la IC, las herramientas disponibles para medirla en la práctica clínica diaria son limitadas y descansan en gran medida en estimaciones a partir de los niveles de creatinina plasmática. Aún cuando el nivel de creatinina permite una apreciación burda del grado de compromiso renal, la estimación de la TFG es la manera más recomendable de evaluar la función renal, pese a las limitaciones inducidas por edad, sexo o superficie corpora ${ }^{19-12}$. La fórmula de Cockroft-Gault, de amplio uso en la clínica, no fue desarrollada para evaluar directamente la TFG sino el clearance de creatinina como indicador vicariante de la filtración glomerular. Por este motivo, esta ecuación tiende a sobrestimar la TFG a medida que se incrementa el grado de disfunción renal. En nuestra población, la estimación de TFG por Cockroft-Gault se asoció a una amplia variabilidad de resultados en relación a los estimados de MDRD-4 o CKD-Epi y su capacidad para predecir eventos fue significativamente menor. Estos resultados contrastan con los reportados por Zamora et al., quienes reivindicaron la utilidad de la fórmula de Cockroft en pacientes con $\mathrm{IC}^{13}$. Sin embargo, en el trabajo mencionado se incluyó una población predominantemente masculina y caucásica; en comparación, nuestra cohorte incluye una elevada proporción de mujeres de etnia hispánica, lo que puede explicar el distinto rendimiento de las ecuaciones.

La fórmula MDRD-4, obtenida a partir de una cohorte de pacientes con falla renal, es poco precisa cuando la TFG supera $60 \mathrm{~mL} / \mathrm{min}$. Nuestra serie muestra resultados similares, con MDRD-4 tendiendo sistemáticamente a sobrevalorar la TFG en pacientes con deterioro incipiente de la función renal. Estos hallazgos son consistentes con los de la literatura, que sugieren que MDRD-4 puede clasificar en forma errónea hasta un 30\% de una cohorte, en particular en individuos con TFG $>60 \mathrm{~mL} / \mathrm{min}^{14}$. Pese a esta limitación, la fórmula MDRD-4 entregó mejor información pronóstica que Cockroft-Gault en nuestra población. De manera similar, la nueva fórmula CKD-Epi se asoció a una baja dispersión de los resultados, tendiendo a subestimar la TFG por encima de $60 \mathrm{~mL} / \mathrm{min}$ en comparación a las otras fórmulas, lo que se asoció a una tasa importante de reclasificación en relación al estadio de función renal estimado por Cockroft-Gault. Cuando se comparó con MDRD-4, CKD-Epi exhibía un alto grado de acuerdo salvo en los estadios de compromiso incipiente de la función renal (2-3a). Al igual que para MDRD-4, la estimación de TFG al alta por CKD-Epi mostró ser un buen predictor de mortalidad al año. Estos resultados ponen en evidencia que una estimación más precisa de la TFG permite identificar subgrupos de pacientes de mayor riesgo, siendo una herramienta útil para el clínico a la hora de decidir el inicio de ARA2/IECA o de ajustar la medicación del paciente en el contexto hospitalario y probablemente también en el contexto ambulatorio, como parece sugerir la evidencia disponible $^{15}$. De particular interés es mencionar que el rol predictivo del deterioro en la TFG es mayor en pacientes con IC y falla sistólica, lo que evidencia la particular importancia del compromiso cardiorenal en los pacientes con compromiso de la función sistólica del ventrículo izquierdo, en los cuales la presencia de falla renal a menudo refleja el impacto del hipodébito en el parénquima renal, a diferencia de los pacientes con función sistólica preservada en los cuales la presencia de deterioro en la función renal suele ser una expresión de las comorbilidades ${ }^{16}$.

El deterioro de la función renal es un elemento clave de la fisiopatología de la IC. En efecto, caídas en la tasas de filtración glomerular son muy frecuentes en contexto de una descompensación aguda de IC y, aun cuando a menudo son transitorias, se asocian a incremento en los tiempos de estadía hospitalaria, así como también incrementan el riesgo de readmisión y el riesgo de muerte post-alta ${ }^{17}$. Los mecanismos responsables del deterioro de la función renal 
durante las hospitalizaciones son complejos y no bien conocidos; probablemente la suma de hipotensión debido a la titulación de vasodilatadores y a reducción del volumen circulante efectivo en contexto de una terapia depletiva enérgica participan en dichos cambios mediante la activación de mecanismos de contra-regulación neurohumoral ${ }^{18}$. Paradójicamente, una terapia depletiva enérgica que logre objetivos de hemoconcentración parece ofrecer beneficios que superan sus potenciales efectos adversos. En efecto, en aquellos pacientes que logran una respuesta depletiva adecuada evidenciada mediante disminución del peso corporal o incrementos de hemoglobina o hematocrito en ausencia de transfusiones, el impacto pronóstico del deterioro de función renal al alta es incierto ${ }^{19}$. De manera similar a lo publicado, en nuestra cohorte la presencia de disfunción renal al alta solo tuvo importancia pronóstica en aquellos pacientes que no alcanzaron objetivos de hemoconcentración; sin embargo, la naturaleza retrospectiva de los datos obliga a revisar con cautela las implicaciones de esta asociación. En efecto, no es claro si acaso la falta de rol pronóstico de la disfunción renal al alta en los individuos que responden a la terapia diurética de manera efectiva se debe a los potenciales efectos beneficiosos de la descongestión, o si simplemente evidencia el hecho que los pacientes que responden más enérgicamente a la terapia depletiva son los pacientes mas sanos, en comparación con aquellos con resistencia a diuréticos que probablemente no alcanzan su peso seco al término de la hospitalización ${ }^{20}$.

En conclusión, nuestros hallazgos sugieren que la incorporación de estimaciones más precisas de la función renal como las proveídas por las ecuaciones MDRD-4 y CKDEpi a la practica clínica habitual permite una mejor caracterización de los pacientes con IC, identificando sujetos de alto riesgo susceptibles de beneficiarse con un seguimiento ambulatorio más estricto. Al mismo tiempo, nuestros datos ponen evidencia la necesidad de interpretar las alteraciones de la función renal en el contexto de la función ventricular del paciente y de su respuesta a la terapia depletiva; en pacientes con buena función sistólica y adecuada respuesta a diuréticos, en particular aquellos que presentan aumentos en hemoglobina o hematocrito al momento del alta sin haber requerido apoyo transfusional (un marcador indirecto de reducción significativa de la sobrecarga de volumen), una fluctuación menor de la TFG reviste poca importancia pronóstica en relación a la misma fluctuación en un paciente con falla sistólica o resistencia a la terapia depletiva.

\section{Referencias:}

1. DAMMAN K, NAVIS G, VOORS AA, ASSELBERGS FW, SMILDE TDJ, CLELAND JGF, et al. Worsening Renal Function and Prognosis in Heart Failure: Systematic Review and Meta-Analysis. J Card Fail. 2007; 13:599-608.

2. GOWANS EM, FRASER CG. Biological variation of serum and urine creatinine and creatinine clearance: ramifications for interpretation of results and patient care. Ann Clin Biochem. 1988; 25: 259-263.

3. LEVEY AS, BOSCH JP, LEWIS JB, GREENE T, ROGERS $\mathrm{N}$, ROTH D. A more accurate method to estimate glomerular filtration rate from serum creatinine: a new prediction equation. Modification of Diet in Renal Disease Study Group. Ann Intern Med. 1999; 130:461-470.
4. COCKCROFT DW, GAULT MH. Prediction of creatinine clearance from serum creatinine. Nephron. 1976; 16:31-41.

5. O'MEARA E, CHONG K, GARDNER R, JARDINE A, NEILLY J, MCDONAGH T. The Modification of Diet in Renal Disease (MDRD) equations provide valid estimations of glomerular filtration rates in patients with advanced heart failure. Eur J Heart Fail. 2006; 8:63-67.

6. STEVENS LA, CORESH J, FELDMAN HI, GREENE T, LASH JP, NELSON RG, et al. Evaluation of the modification of diet in renal disease study equation in a large diverse population. J Am Soc Nephrol. 2007; 18:2749-2757. 
7. LEVEY AS. A New Equation to Estimate Glomerular Filtration Rate. Ann Intern Med. 2009; 150:604-612.

8. MANZANO-FERNÁNDEZ S, FLORES-BLANCO P, PÉREZ-CALVO J, RUIZ-RUIZ F, CARRASCO-SÁNCHEZ F, MORALES-RULL J, et al. Comparison of risk prediction with the CKD-EPI and MDRD equations in acute decompensated heart failure. J Card Fail. 2013; 19:583-591.

9. LEVEY AS, CORESH J, BALK E, KAUSZ AT, LEVIN A, STEFFES MW, et al. National Kidney Foundation practice guidelines for chronic kidney disease: evaluation, classification, and stratification. Ann Intern Med. 2003; 139:137-147.

10. BOERRIGTER G, HOCHER B, LAPP H. Changes in Renal Function in Congestive Heart Failure. Curr Heart Fail Rep. 2013; 10:285-295.

11. OH J, KANG S-M, HONG N, YOUN J-C, HAN S, JEON E-S, et al. Registry OBOTK. The CKD-EPI is more accurate in clinical outcome prediction than MDRD equation in acute heart failure: Data from the Korean Heart Failure (KorHF) Registry. Int J Cardiol. 2013; 167:1084-1087.

12. LEVEY AS, DE JONG PE, CORESH J, NAHAS ME, ASTOR BC, MATSUSHITA K, et al. The definition, classification, and prognosis of chronic kidney disease: a KDIGO Controversies Conference report. Kidney Int. 2010; 80:17-28.

13. ZAMORA E, LUPÓN J, VILA J, URRUTIA A, DE ANTONIO M, SANZ H, et al. Estimated glomerular filtration rate and prognosis in heart failure. JAC. 2012; 59:1709-1715.

14. FROISSART M. Predictive Performance of the Modification of Diet in Renal Disease and Cockcroft-Gault Equations for
Estimating Renal Function. J Am Soc Nephrol. 2005; 16:763773.

15. MCALISTER FA, EZEKOWITZ JA, TARANTINI L, SQUIRE I, KOMAJDA M, BAYES-GENIS A, et al. Renal dysfunction in heart failure patients with preserved versus reduced ejection fraction: Impact of the new CKD-EPI formula. Circ Heart Fail. 2012; 5:309-14

16. SCRUTINIO D, PASSANTINO A, SANTORO D, CATANZARO R. The cardiorenal anaemia syndrome in systolic heart failure: prevalence, clinical correlates, and long-term survival. Eur J Heart Fail. 2010; 13:61-67.

17. LOGEART D, TABET J-Y, HITTINGER L, THABUT G, JOURDAIN P, MAISON P, et al. Transient worsening of renal function during hospitalization for acute heart failure alters outcome. Int J Cardiol. 2008; 127:228-232.

18. FORMAN DE, BUTLER J, WANG Y, ABRAHAM WT, O'CONNOR CM, GOTTLIEB SS, et al. Incidence, predictors at admission, and impact of worsening renal function among patients hospitalized with heart failure. J Am Coll Cardiol. 2004; 43:61-67.

19. TESTANI JM, CHEN J, MCCAULEY BD, KIMMEL SE, SHANNON RP. Potential effects of aggressive decongestion during the treatment of decompensated heart failure on renal function and survival. Circulation. 2010; 122:265-272.

20. NEUBERG GW, MILLER AB, O'CONNOR CM, BELKIN RN, CARSON PE, CROPP AB, et al. Prospective randomized amlodipine survival evaluation. Diuretic resistance predicts mortality in patients with advanced heart failure. Am Heart J. 2002; 144:31-38. 